CLINICAL STUDY

\title{
Five cases with central diabetes insipidus and hypogonadism as first presentation of neurosarcoidosis
}

Catharina Bullmann, Michael Faust, Axel Hoffmann, Christina Heppner ${ }^{1}$, Friedrich Jockenhövel, Dirk MüllerWieland and Wilhelm Krone

Klinik II und Poliklinik für Innere Medizin der Universität zu Köln, Cologne, Germany and ${ }^{1}$ NIH/NIDDK, Bethesda, Maryland, USA

(Correspondence should be addressed to Catharina Bullmann, Klinik II und Poliklinik für Innere Medizin, Universität zu KöIn, Joseph-Stelzmann-Str. 9 , 50924 Cologne, Germany; Email: ai005@uni-koeln.de)

\begin{abstract}
Objectives: We retrospectively reviewed 5 patients with neurosarcoidosis, who all presented with central diabetes insipidus and hypogonadism.

Design: This was a single-centre, retrospective analysis of 5 cases with a minimum follow-up of 2 years. Methods: Case analysis included clinical, biochemical, and endocrinological evaluation and frequent CT/MRI scans of involved organs as primary evaluation and in response to immunosuppressive therapy.

Result: Neurosarcoidosis was diagnosed in all patients. Two patients had no proven extracerebral manifestation and had a stable disease over 3 and 5 years. One patient showed deterioration with corticosteroids alone but partial remission after additional cyclophosphamide. Pituitary dysfunction remained unchanged in all patients, despite total clinical and radiological remission in two patients. However, one of these patients died of acute granulomatous meningoencephalitis after two years of follow-up.

Conclusion: Although the presenting symptoms of neurosarcoidosis may vary, the occurrence of central diabetes insipidus associated with typical radiological features is suggestive of neurosarcoidosis. However, there is an increasing number of case reports on lymphocytic hypophysitis. Without the bioptic diagnosis, the differentiation between potentially lethal isolated neurosarcoidosis and lymphocytic hypophysitis is difficult. These cases demonstrate the difficulties in diagnosing neurosarcoidosis and reflect experiences with follow-up parameters.
\end{abstract}

European Journal of Endocrinology 142 365-372

\section{Introduction}

Neurosarcoidosis and lymphocytic hypophysitis are important differential diagnoses of central diabetes insipidus (CDI) caused by inflammatory diseases, and are difficult to diagnose. Sarcoidosis is a multisystem disease of unknown aetiology. Involvement of the central nervous system is a well-known manifestation of the disease occurring in approximately 5\% of patients, and precedes additional symptoms in 30\% of cases (1).

CDI occurs in about $25 \%$ of patients with neurosarcoidosis (2). Therefore, hypothalamic and pituitary sarcoidosis is well known (3), although an isolated effect on the pituitary gland is rare (4).

Lymphocytic hypophysitis is a rare autoimmune disorder of the pituitary with a striking preference for females in the postpartum period $(5,6)$. The presenting symptoms include headache, visual symptoms and signs of anterior pituitary insufficiency in $60 \%$ of cases and CDI in 20\% of cases $(5,7)$. An associated autoimmune disease is found in about $25 \%$ of cases.

In this report we describe five patients who initially presented with central diabetes insipidus and hypogonadism. Clinical evaluation led to the diagnosis of neurosarcoidosis in all the patients.

\section{Patients and methods}

Two women and three men in the age range 21-41 years at first presentation were referred to our department. Primary diagnostic evaluation was performed by our department in all patients except patient (pt) 4 .

\section{Laboratory investigations}

Routine laboratory investigations were performed in all patients, erythrocyte sedimentation rate (ESR) in 4 patients, serum angiotensin converting enzyme activity (ACE) and soluble interleukin 2 receptor (IL-2R) level in 
Table 1 Clinical, radiological results and extraneurologic manifestation.

\begin{tabular}{|c|c|c|c|c|c|}
\hline & \multicolumn{5}{|c|}{ Patient } \\
\hline & $1(f)$ & $2(\mathrm{f})$ & $3(\mathrm{~m})$ & $4(m)$ & $5(m)$ \\
\hline $\begin{array}{l}\text { Age at first presentation (years) } \\
\text { Duration of polyuria* (months before initial }\end{array}$ & 40 & 41 & 27 & 21 & 26 \\
\hline $\begin{array}{l}\text { presentation) } \\
\text { Additional symptoms at first presentation }\end{array}$ & $\begin{array}{c}12 \\
\text { Amenorrhoea, } \\
\text { fatigue }\end{array}$ & $\stackrel{5}{\text { Amenorrhoea }}$ & $\begin{array}{c}3 \\
\text { Impotence }\end{array}$ & $\begin{array}{c}2 \\
\text { Impotence }\end{array}$ & $\begin{array}{c}3 \\
\text { Visual impairment, } \\
\text { loss of secondary } \\
\text { hair coat }\end{array}$ \\
\hline Extracerebral manifestation & None & Hepatopathia? & $\begin{array}{l}\text { Parahilary } \\
\text { lymph nodes, } \\
\text { muscular, } \\
\text { peritoneal (?) }\end{array}$ & $\begin{array}{l}\text { Eye, gastrointestinal, } \\
\text { skin, lymph nodes }\end{array}$ & $\begin{array}{l}\text { Lung, parahilary } \\
\text { lymph nodes, } \\
\text { epicard }\end{array}$ \\
\hline $\begin{array}{l}\text { Chest X-ray (enlargement of lymph nodes) } \\
\text { CT chest (enlargement of lymph nodes) } \\
\text { Brain MRI }\end{array}$ & $\begin{array}{l}\text { Negative } \\
\text { Negative }\end{array}$ & $\begin{array}{l}\text { Negative } \\
\text { Negative }\end{array}$ & $\begin{array}{l}\text { Negative } \\
\text { Positive }\end{array}$ & $\begin{array}{l}\text { Positive } \\
\text { Positive }\end{array}$ & $\begin{array}{l}\text { Positive } \\
\text { Positive }\end{array}$ \\
\hline $\begin{array}{l}\text { Enhancement of pituitary stalk } \\
\text { Histology }\end{array}$ & $\begin{array}{l}\text { Yes } \\
\text { nd }\end{array}$ & $\begin{array}{l}\text { Yes } \\
\text { nd }\end{array}$ & $\begin{array}{c}\text { Yes } \\
\text { Transbronchial }\end{array}$ & $\begin{array}{l}\text { Yes } \\
\text { Cervical lymph node }\end{array}$ & $\begin{array}{c}\text { No } \\
\text { Transbronchial }\end{array}$ \\
\hline
\end{tabular}

$\mathrm{m}=\mathrm{male} ; \mathrm{f}=$ female; $\mathrm{nd}=$ not determined; * intake exceeds $50 \mathrm{ml} / \mathrm{kg}$ body weight.

all patients. Tests were performed for tuberculosis, for syphilis in 4 patients (pt $1-3,5$ ), for histiocytosis $X$ in 3 (pt 1-3) and cerebrospinal fluid (CSF) was evaluated in patient 3. Pituitary functions were tested using $100 \mu \mathrm{g}$ corticotropin-releasing hormone (CRH) (Ferring, Kiel, Germany) and $100 \mu \mathrm{g}$ gonadotropin-releasing hormone (GnRH) (Hoechst, Frankfurt, Germany) (8) following standard protocols. Complete or partial central diabetes insipidus was diagnosed by standard water deprivation test followed by i.v. administration of $2 \mu \mathrm{g}$ desamino-8D-arginine vasopressin (DDAVP) (9). Partial or complete remission was defined by resolution

Table 2 Laboratory results.

\begin{tabular}{|c|c|c|c|c|c|}
\hline & \multicolumn{5}{|c|}{ Patient } \\
\hline & $1(\mathrm{f})$ & $2(f)$ & $3(\mathrm{~m})$ & $4(\mathrm{~m})$ & $5(\mathrm{~m})$ \\
\hline $\operatorname{ESR}(\mathrm{mm} / \mathrm{h})$ & 22 & 43 & 33 & nd & 11 \\
\hline ACE $(50-127 \mathrm{U} / \mathrm{l})$ & 155 & 133 & 45 & 35 u.th. & 166 \\
\hline Soluble IL-2R $(<900 \mathrm{U} / \mathrm{I})$ & 494 & 500 & $690 \rightarrow 2124$ & 1410 u.th. & 575 \\
\hline $\mathrm{TSH}(0.23-4.0 \mathrm{mU} / \mathrm{l})$ & 0.28 & 0.1 & 0.7 & 2.5 u.th. & 1.7 \\
\hline $\mathrm{fT}_{3}(4.6-9.2 \mathrm{pmol} / \mathrm{l})$ & 5.8 & 5.1 & 3.1 & 3.5 u.th. & 5.2 \\
\hline $\mathrm{fT}_{4}(10.2-25.7 \mathrm{pmol} / \mathrm{l})$ & 14.1 & 20.5 & $<9$ & $<9$ u.th. & $<9$ \\
\hline Prolactin (f: $67-696 \mathrm{mlU} / \mathrm{l} ; \mathrm{m}: 50-424 \mathrm{mlU} / \mathrm{l})$ & 784 & 792 & 1125 & 1144 & 384 \\
\hline $17 \beta$ Estradiol (36-734 pmol/l) & 73 & $<36$ & - & - & - \\
\hline Testosterone $(10.4-48.5 \mathrm{nmol} / \mathrm{l})$ & - & - & 0.8 & 6.0 & 3.5 \\
\hline GH basal $(<8.6 \mu \mathrm{g} / \mathrm{l})$ & 0.9 & 1.0 & 1.1 & 0.2 & $<0.3$ \\
\hline IGF-I (f: $119-333 \mu \mathrm{g} / \mathrm{l}, \mathrm{m}: 126-460 \mu \mathrm{g} / \mathrm{l})$ & 135 & 132 & 242 & 290 & 160 \\
\hline GnRH test & & & & u.th. & \\
\hline LH basal (m: 1.3-12.9; f: *IU/I) & 2.2 & 0.6 & $<0.5$ & $<0.1$ & $<0.5$ \\
\hline LH peak (IU/I) & 16.2 & 7.4 & $<0.5$ & 0.2 & $<0.5$ \\
\hline FSH basal (m: 0.9-15.0; f: *IU/l) & 7.8 & 3.9 & $<0.5$ & 0.7 & 0.7 \\
\hline FSH peak (IU/l) & 16.4 & 6.9 & 0.6 & 0.4 & 1.5 \\
\hline $\mathrm{CRH}$ test & & & & u.th. & \\
\hline Cortisol basal $(115-356 \mathrm{nmol} / \mathrm{l})$ & 322 & 148 & 400 & 223 & 605 \\
\hline Cortisol peak (nmol/l) & 612 & 422 & 673 & 524 & 204 \\
\hline Plasma ACTH basal $(1.98-11.2 \mathrm{pmol} / \mathrm{l})$ & 5.8 & 3.1 & 1.0 & 5.4 & 3.1 \\
\hline Plasma ACTH peak (pmol/l) & 16 & 16 & 11 & 8.8 & 48 \\
\hline \multicolumn{6}{|l|}{ Water deprivation test } \\
\hline Urine osmolality maximal (mmol/kg) & 540 & 498 & 418 & n.a. & 135 \\
\hline Body weight reduction (\%) & 4.4 & 3 & 2.2 & n.a. & 2.7 \\
\hline
\end{tabular}

Parameters determined in serum if not indicated otherwise; normal values are given in parentheses; $m=$ male; $f=$ female; ${ }^{*}=$ female normal values dependent to menstrual cycle; $\mathrm{TSH}=$ thyrotrophin; $\mathrm{fT}_{3}=$ free tri-iodothyronine; $\mathrm{fT}_{4}=$ free thyroxine; $\mathrm{GH}=$ growth hormone; IGF-I = insulin-like growth factor-I; $\mathrm{LH}=$ luteinising hormone; $\mathrm{FSH}=$ follicle-stimulating hormone; nd = not determined; u.th. = under therapy, n.a. $=$ not available. 
of clinical findings, brain magnetic resonance tomography (MRI) findings and enlarged lymph nodes in computed tomography (CT) thorax (partial or complete) respectively.

\section{Imaging studies}

Brain MRI with and without gadolinium was performed in all patients initially and during follow-up. X-ray and CT with and without contrast were carried out in all patients to detect or exclude pulmonary manifestation and involvement of hilary lymph nodes in sarcoidosis respectively. In patients $1-3$ tests included X-ray of the skull and both femurs to exclude histiocytosis.

\section{Case reports}

\section{Symptoms and findings at initial evaluation}

Symptoms, and radiological and histological findings are summarised in Table 1. Results of laboratory investigations and endocrine parameters are given in Table 2. Patients presented with central diabetes insipidus and hypogonadism of 1 to 12 months duration. None of the patients reported fever or chills at first presentation. All patients except patient 4 were seen at initial presentation without immunosuppressive therapy.

Abnormal laboratory parameters included elevated ESR in 3, elevated serum ACE in 3, and pathological IL-2R levels in 1 patient. Tuberculosis, syphilis and histiocytosis $\mathrm{X}$ could be ruled out in patients $1-3$. Water deprivation test revealed a partial to total central diabetes insipidus in all patients, all responded to desmopressin with an increased urine osmolality (9). Further endocrine evaluation documented elevated prolactin levels in all patients with mainly pituitary involvement. Patient 2 showed additional secondary thyroidal insufficiency and all but patient 1 showed secondary/tertiary hypogonadism. The mildly elevated prolactin level in patient 1 may also account for the secondary amenorrhoea of 12 months duration. Interestingly, patient 5 showed an overshoot reaction of adrenocorticotropin (ACTH) and below normal reaction of cortisol to $\mathrm{CRH}$ stimulation, which may account for the mainly hypothalamic involvement also demonstrated in brain MRI, or an adrenal insufficiency (see Fig. 5a) (10).

Ophthalmological evaluation revealed impaired vision of the right eye in patient 5. Brain MRI showed enhancement of the pituitary stalk with gadolinium in all but one patient (pt 5). However, patient 5 had multiple cerebral lesions. Beside pituitary stalk involvement, patient 4 had four additional lesions and patient 3 indicated discreet brainstem and cerebellar lesions. Cases 1 and 2 showed elevated chiasma opticum. X-ray and CT of the chest revealed enlarged hilary lymph nodes in patients 4 and 5 .

\section{Clinical course and therapy}

After exclusion of the differential diagnosis, the combination of clinical, laboratory and radiological tests led to diagnosis of neurosarcoidosis. In all cases therapy was started with prednisone $(0.5-1.5 \mathrm{mg} / \mathrm{kg}$ body weight). Further treatment was modified according to clinical progress. All patients were supplemented with desmopressin, while gonadal and thyroid dysfunction were corrected by the usual substitutional therapy $(9,11)$.

Despite good response to immunosuppressive therapy as assessed by radiological parameters or a stable disease, no patient recovered from central diabetes insipidus and only patient 1 recovered from associated gonadal insufficiency.

Patient 1 The patient presented with central diabetes insipidus, secondary amenorrhoea and thickening of the pituitary stalk. Due to an elevated ACE level and the enhancement of the pituitary stalk, the diagnosis of isolated neurosarcoidosis was presumed. Prednisone therapy over a course of 12 weeks was started, and ESR and ACE were normalised in response. Radiologically, enhancement of the pituitary stalk diminished. During the follow-up of 36 months, normal menstrual cycles were re-established while diabetes insipidus persisted (Fig. 1).

Patient 2 This patient showed similar findings to patient 1 with additional secondary/tertiary thyroid insufficiency leading to a diagnosis of isolated neurosarcoidosis. The patient was treated with prednisone for four years. Elevated ACE levels and previously pathologic hepatic parameters (ASAT, ALAT) decreased to within the normal range. After reducing the prednisone dosage, ACE level remained normal but ASAT and ALAT rose again into the pathological range. Viral, autoimmune and metabolic causes were ruled out. While ASAT and ALAT normalised without any steroid therapy, pituitary dysfunction and radiological results remained unchanged over the course of five years (Fig. 2).

Patient 3 The patient presented with central diabetes insipidus and hypogonadism at our department. Because serum levels of ACE as well as soluble IL-2R were normal, and chest X-ray showed no pathological alterations, the patient was not started on steroids initially. During the clinical course, the patient developed cerebellar symptoms. Further diagnostic tests, including chest CT, revealed enlarged hilary lymph nodes. CSF showed unspecific findings consistent with inflammatory changes. Diagnosis of sarcoidosis was confirmed by biopsy of the lymph nodes, and IL-2R levels increased into the pathological range. However, even under corticosteroids the patient developed further progression of the disease with recurrent cerebral 

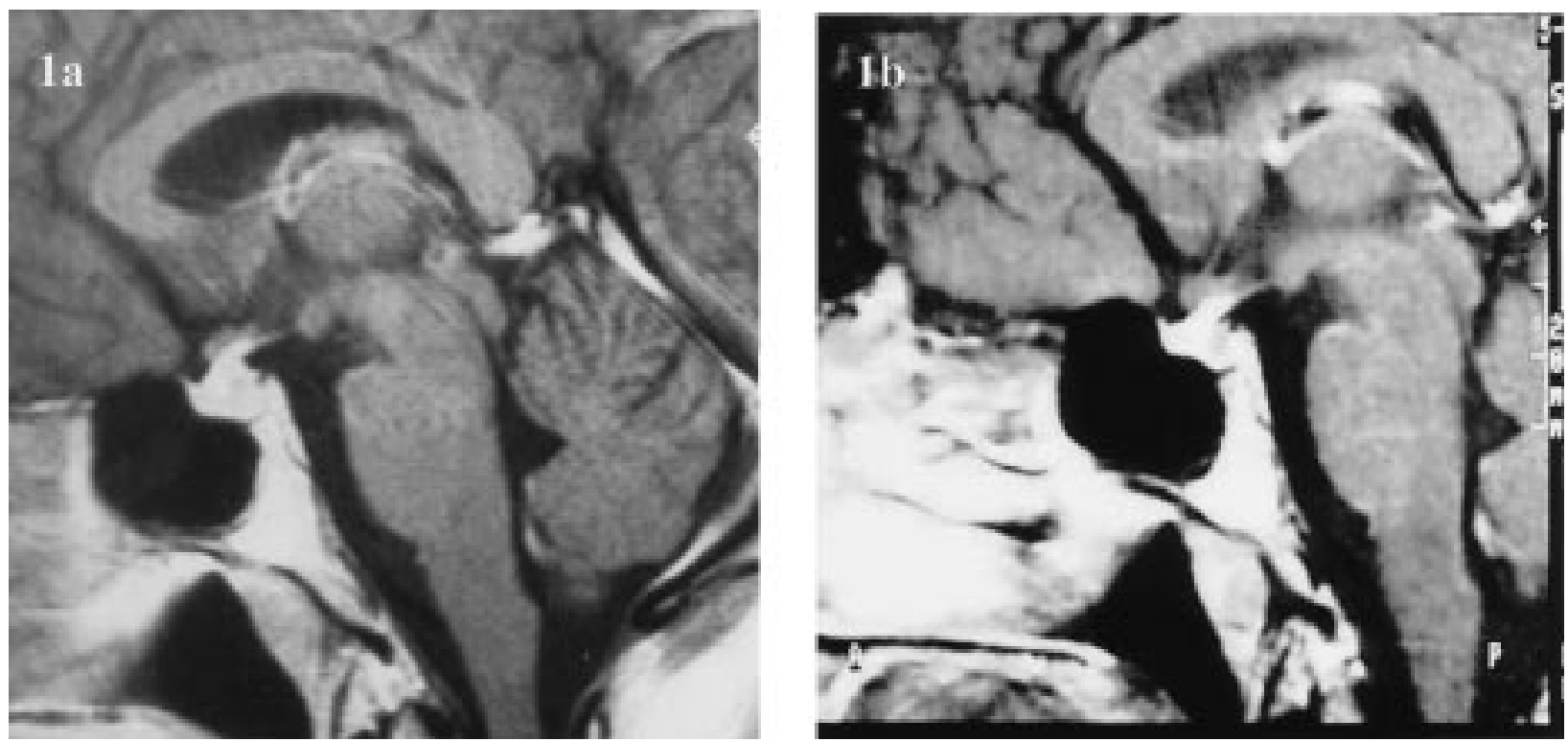

Figure 1 Saggital brain T1-weighted MR image after i.v. gadolinium demonstrates (a) an enhancing mass of the pituitary and the pituitary stalk in patient 1. (b) Status post corticosteroid treatment with a small decrease in the size of the enhancing mass.

oedema, even after implantation of a ventricular shunt. The cerebellar and muscular involvement led to severe handicap in daily activities. Escalation of immunosuppressive therapy by means of $150 \mathrm{mg}$ daily cyclophosphamide (12) resulted in impressive clinical improvement, with ESR and IL-2R levels decreased into the normal range. Throughout the follow-up of one year steroids could be decreased to $1 \mathrm{mg}$ dexamethasone preventing further aggravation of steroid side effects. Daily cyclophosphamide therapy was continued for nine months, thereafter it was changed to pulse treatment with a four-week interval (Fig. 3).

Patient 4 The patient presented with a history of central diabetes insipidus for two months and hypogonadism for nine months. Brain MRI showed enhancement of
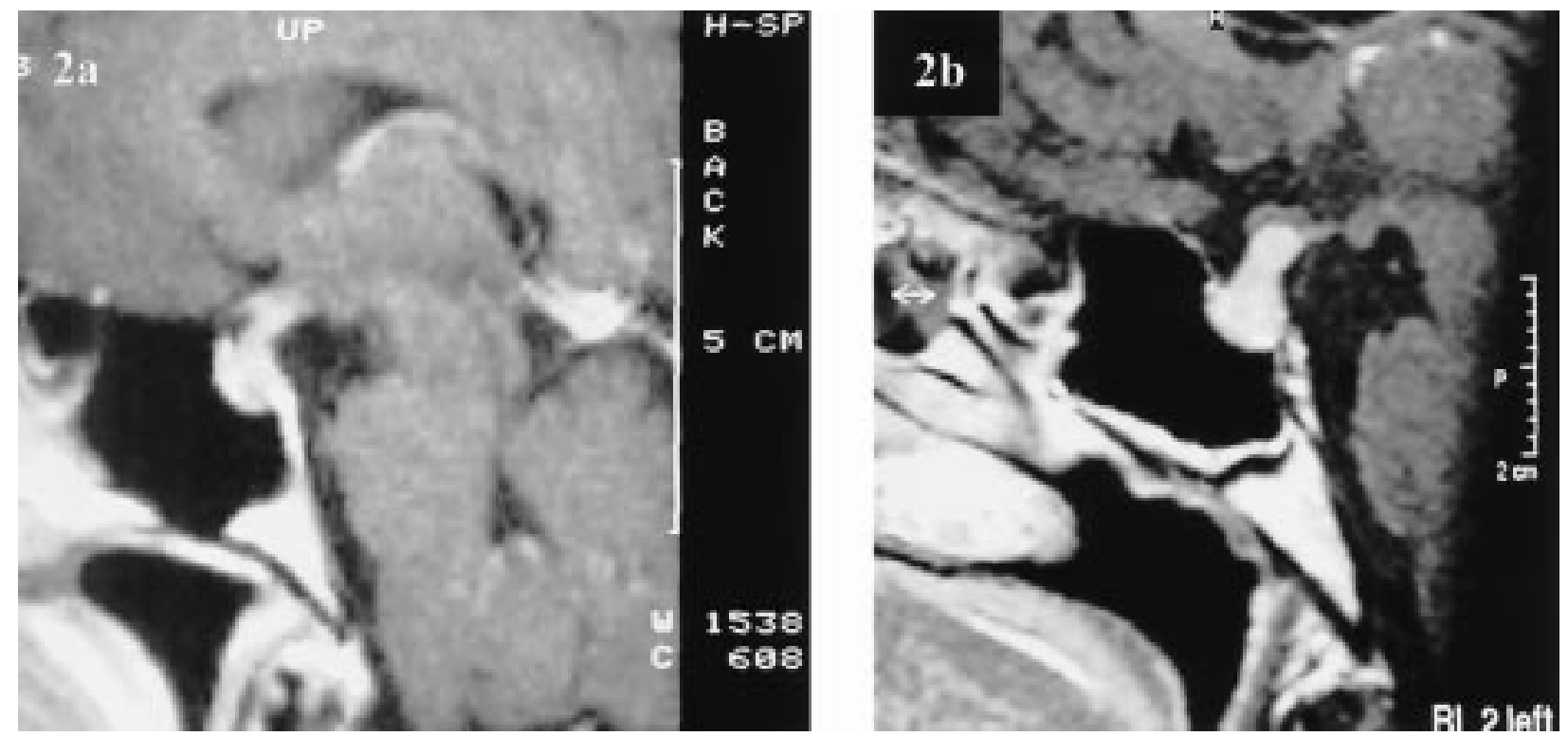

Figure 2 Saggital brain T1-weighted MR image after i.v. administration of gadolinium demonstrates (a) an enhancing mass of the pituitary and enhancing pituitary stalk in patient 2 , and (b) no significant change after corticosteroid treatment. 

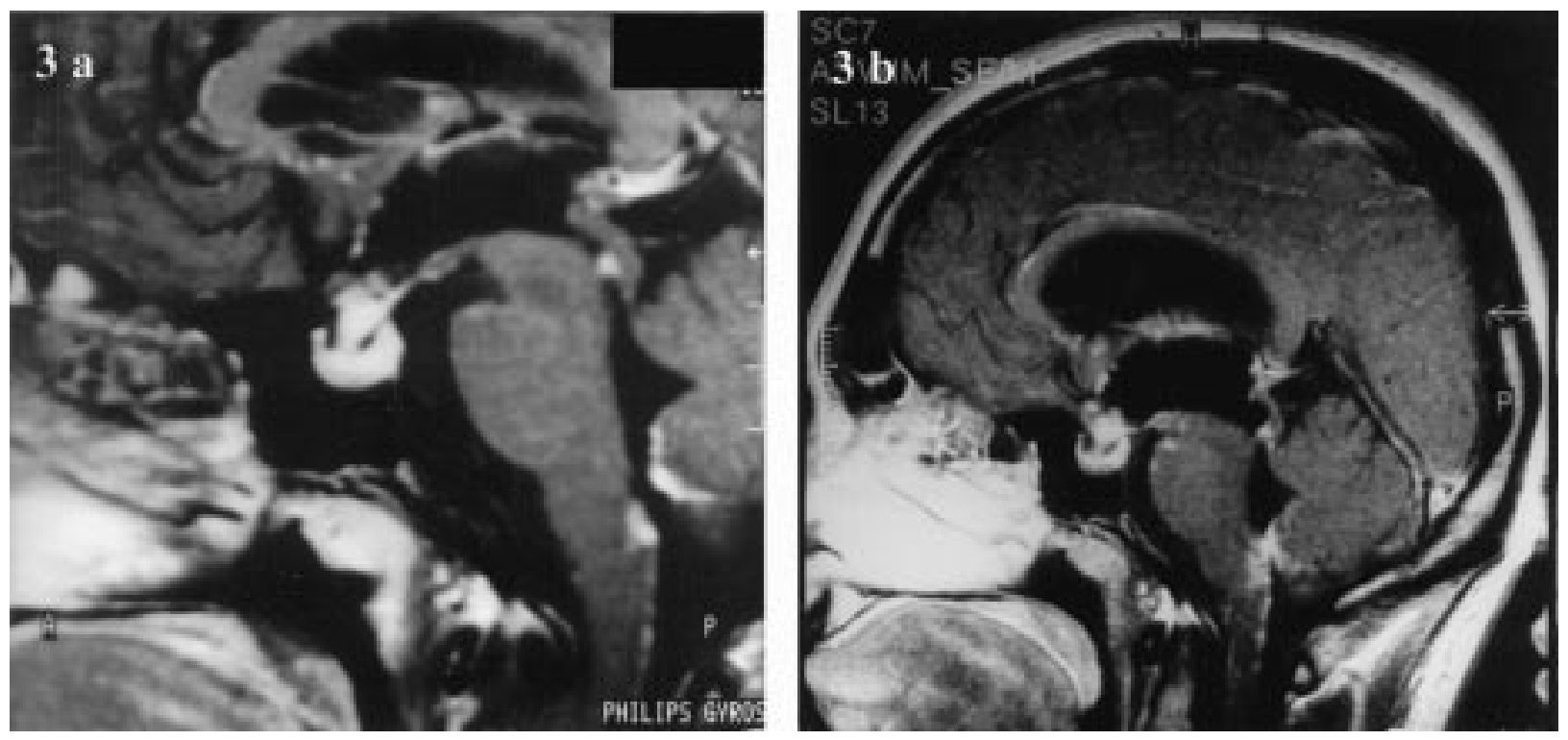

Figure 3 Saggital brain T1-weighted MR image after i.v. administration of gadolinium shows (a) an enhancing mass within the pituitary stalk with additional enhancement at the base of the fourth ventricle referring to brainstem and cerebellum at initial presentation in patient 3 , and (b) development of hydrocephalus during follow-up leading to implantation of a ventricular shunt one year after follow-up despite corticosteroid treatment.

pituitary stalk. Generalised sarcoidosis was suspected because of enlarged bihilary and abdominal lymph nodes in the CT scan. Histological proof was obtained from a neck lymph node. Prednisone therapy over three years resulted in good remission of pituitary, bihilary and abdominal manifestation measured radiologically, although central diabetes insipidus and hypogonadism

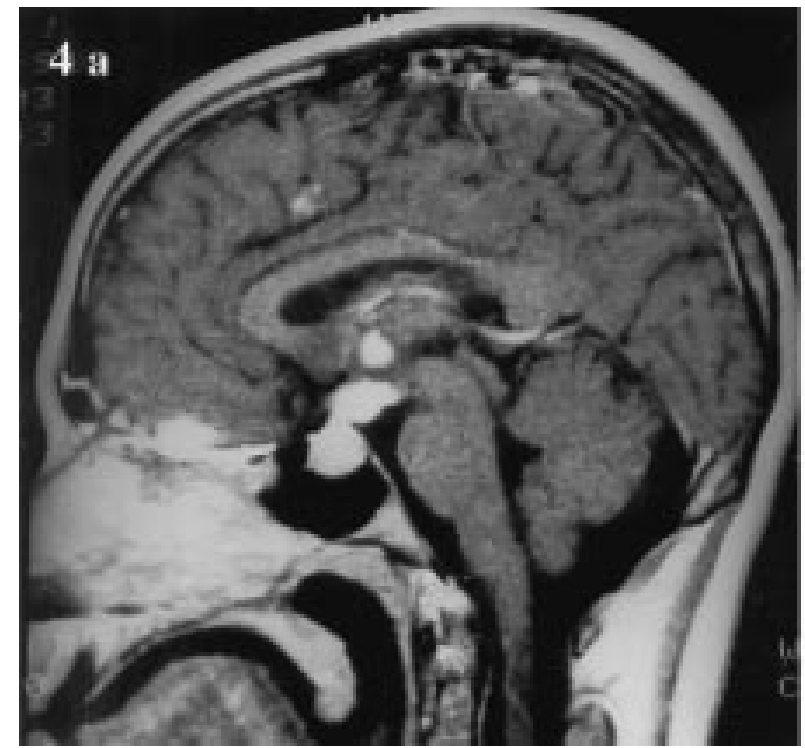

persisted. After discontinuation of steroid therapy, organ manifestation remained stable for two years, but histologically proved skin involvement was observed (Fig. 4).

Patient 5 Clinically, the patient showed central diabetes insipidus, hypogonadism and visual impairment.

Figure 4 Saggital brain T1-weighted MR image after i.v. administration of gadolinium shows (a) an enhancement of the pituitary and the pituitary stalk and additional enhancement within the frontal lobe and the thalamus at initial presentation in patient 4 and (b) complete regression in response to steroid therapy after five years of follow-up.

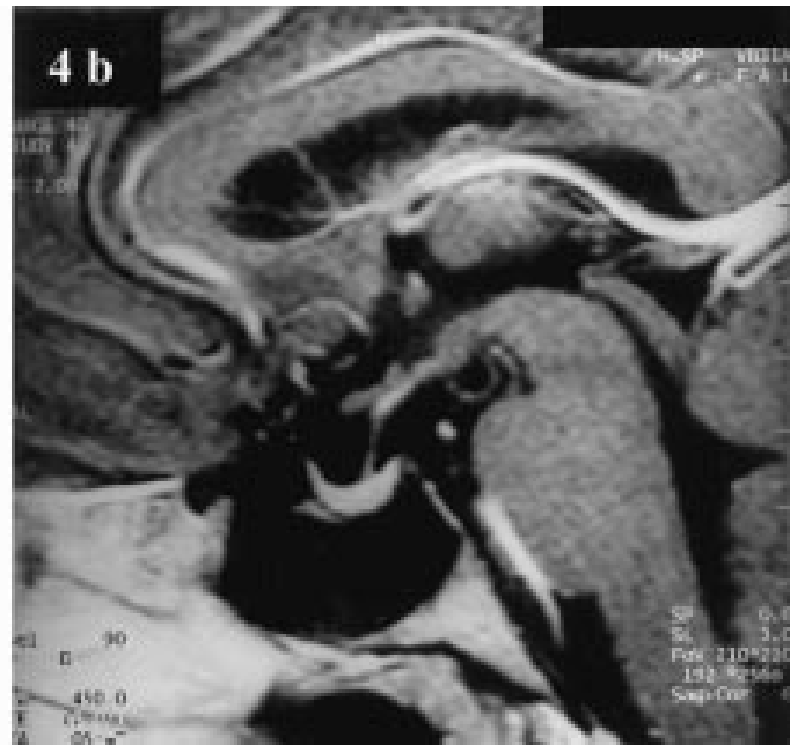



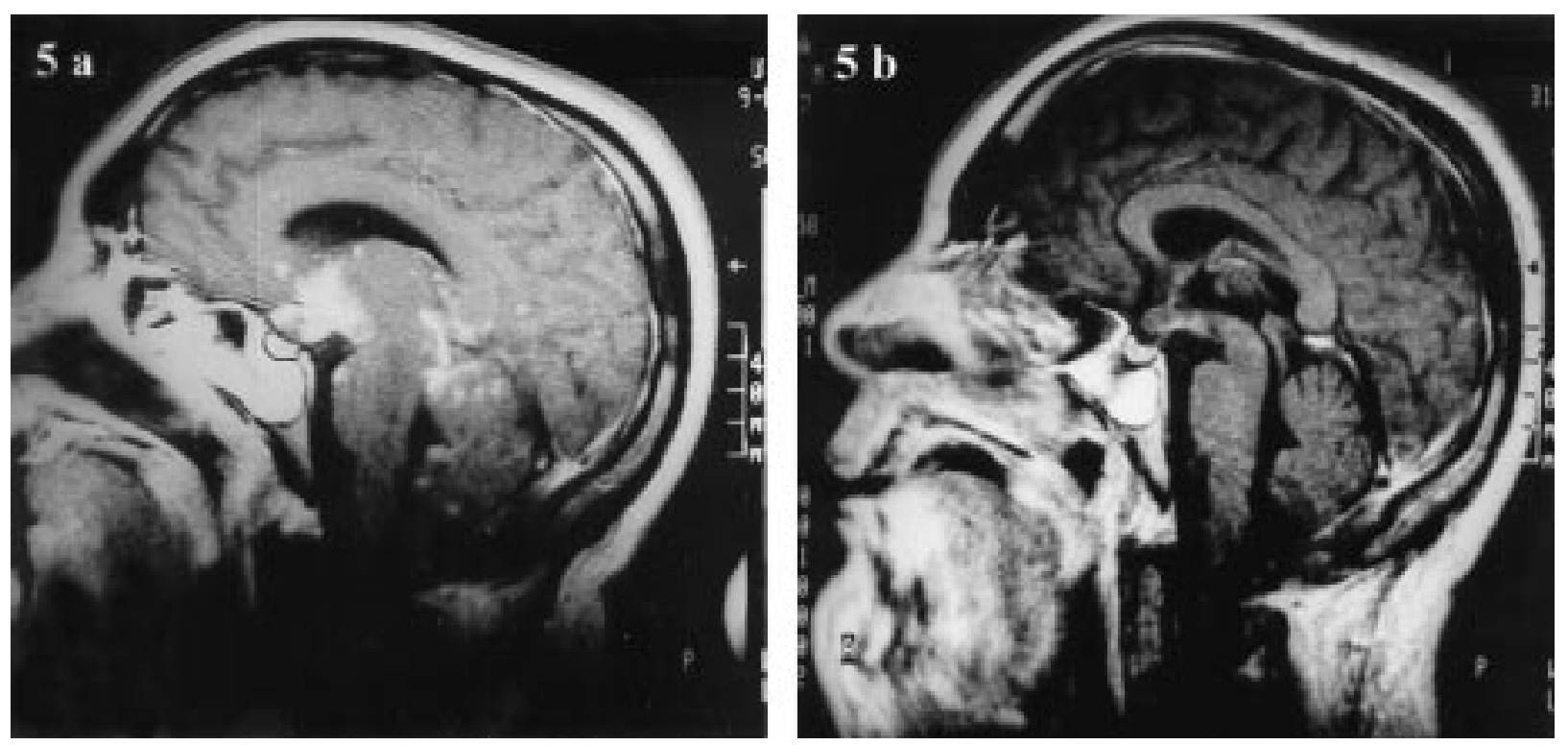

Figure 5 Saggital brain T1-weighted MR image after i.v. administration of gadolinium shows (a) multilocular enhancement predominating in the thalamus, the chiasma opticum and meningeal enhancement at initial presentation in patient 5 and (b) complete regression after 18 months of follow-up in response to steroid therapy.

Already at first presentation generalised sarcoidosis was suspected due to elevated ACE levels, severe involvement of the optic nerve and the hypothalamus in brain MRI, and enlarged bihilary lymph nodes in chest CT. Biopsy of the lymph nodes revealed granulomatous changes. After starting prednisone treatment, clinical improvement was seen associated with a decrease in ACE levels into the normal range. Corticosteroids were tapered over a period of two years to $10 \mathrm{mg}$ methylprednisone. Despite improvement in brain MRI, pituitary dysfunction remained unchanged (Fig. 5b). Rapid and unexpected deterioration of the disease led to an acute admission, but intensive care treatment could not prevent the death of the patient two years after initial diagnosis due to severe meningoencephalitis together with cerebral oedema. Post-mortem granulomatous alterations of the cerebrum (hypothalamus, chiasma, midbrain, cerebellum and epiphysis) with partial conglomerate tumorous changes were found. Lung, parahilary lymph nodes and right epicard showed additional granulomatous involvement.

\section{Discussion}

Little is known about the incidence and course of isolated neurosarcoidosis. Involvement of the neuromuscular system is seen in 5\% of patients with sarcoidosis. Incidence of subclinical neurosarcoidosis and neuromuscular manifestation detected in pathological studies appears to be higher $(1,13)$. In about $30 \%$ of these cases, involvement of the neuromuscular system appeared to be the presenting symptom. Delay of appropriate treatment is the major problem in the care of the patient.

We have described five patients with endocrine alterations as the first clinical manifestation of neurosarcoidosis. Two of our patients had no proven extraneurological manifestation, while development of pituitary dysfunction led to the diagnosis of multi-organ involvement in the other three cases. Chapelon and colleagues found $6 / 30$ cases with isolated neurosarcoidosis, mostly presenting with myopathy or polyneuropathy but only one with endocrine dysfunction alone (diabetes insipidus and hypogonadism) (1). Two of our patients had no signs of systemic sarcoidosis. Although there was no histological proof for the diagnosis, neurosarcoidosis was most likely because of elevated ESR and ACE levels. To our knowledge only five other cases have been described with a similar history (14-19).

Neurosarcoidosis is a difficult differential diagnosis of central diabetes insipidus and should be considered by the endocrinologist. The disease is associated with intrathoracic lesions in about $70 \%$ of cases; therefore, an intensive search for enlarged hilary lymph nodes should be performed (1). While two of our patients did have pathological chest X-ray and chest CT, one showed a normal chest X-ray but enlarged lymph nodes in chest CT later in the clinical course. In 1986, Oksanen demonstrated that normal chest X-ray at initial presentation does not exclude generalised sarcoidosis (2). As seen in patient 3, normal chest X-ray does not exclude enlarged mediastinal lymph nodes. Therefore, careful follow-up and an intensive search for systemic 
sarcoidosis including chest CT is mandatory in patients with presumed isolated neurosarcoidosis of the pituitary.

ESR and serum calcium may help in diagnostic work but are unspecific parameters. Three of our patients showed moderately elevated ESR. Occasionally, an elevated serum calcium level is documented with isolated neurosarcoidosis (20). Laboratory parameters with the highest specificity and sensitivity for detecting sarcoidosis are ACE level and soluble IL-2R level in serum $(21,22)$. ACE is produced in the sarcoid granuloma by immigrated macrophages and is thought to reflect the granuloma load. The soluble IL-2R is produced by T-lymphocytes and therefore reflects the specific T-cell response of the inflammation. In general, serum ACE is known to have a sensitivity of $55-60 \%$ and a specificity of $25 \%$ for the detection of sarcoidosis, but is dependent on the extent of the lesions (23). While the serum ACE level was elevated in both cases of presumed isolated neurosarcoidosis, IL-2R levels showed normal values in these cases. The soluble IL-2R level is known to have the same specificity but higher sensitivity (83\%) than the serum ACE level in pulmonary sarcoidosis and, furthermore, it appears to be an indicator of disease progression $(24,25)$. In case 3 , normal IL-2R levels increased at the time when systemic sarcoidosis was diagnosed and decreased into the normal range, correlating with a good clinical immunosuppressive response. Increased CSF-ACE levels usually represent meningeal involvement in neurosarcoidosis (20). Patient 3 had questionable meningeal involvement but normal CSF-ACE levels. About half of the patients with neurosarcoidosis show pleocytosis and elevated protein levels in CSF (2), as in infectious meningitis, which was excluded in our case. All CSF findings are characteristic of cerebrospinal tract involvement and are not explained by a leakage through the blood-brain barrier (13). Elevated serum ACE and soluble IL-2R levels at the time of diagnosis seem to be a valid follow-up parameter.

Brain MRI is the most sensitive imaging procedure for detecting cerebral sarcoidosis and its response to steroid therapy. Periventricular distribution of lesions and leptomeningeal enhancement are the two most common abnormal findings in neurosarcoidosis (60 and $65 \%$ ). The latter is especially helpful in discriminating neurosarcoidosis from multiple sclerosis and lymphocytic hypophysitis (Fig. 3). Thickening of the pituitary stalk may be detected, together with endocrine symptoms $(26,27)$. All of our patients with pituitary involvement showed an enlargement of the pituitary stalk (cases 1, 2, 3, 4). In cases 1 and 2 this was the only radiological abnormality.

Regarding the two female patients with isolated pituitary neurosarcoidosis, lymphocytic hypophysitis $(\mathrm{LyH})$ is an important differential diagnosis. Since 1961, around 100 case reports of $\mathrm{LyH}$ have been documented, with increasing frequency $(5,6)$. There is no specific laboratory parameter for evaluating the diagnosis. Therefore, case reports document occasional findings from autopsies or pituitary surgery (6). Our two female patients showed no correlation with the postpartum period or associated autoimmune endocrine disease. Without the possibility of a biopsy on the pituitary, exclusion of the differentials leads the diagnostic approach. The difficult differentiation between $\mathrm{LyH}$ and isolated neurosarcoidosis and the knowledge that even $\mathrm{LyH}$ may respond to steroid therapy, should lead to immunosuppressive therapy in case any parameter is positive for neurosarcoidosis. However, efficacy of corticosteroid therapy remains uncertain in the case of LyH (5). Pituitary adenoma was unlikely in all cases because diabetes insipidus was a presenting symptom, and it was excluded by the unusual radiological picture and the endocrine parameters. Histiocytosis includes a spectrum of benign and malignant diseases, consisting of unifocal or disseminated bone lesions in young patients and possible other organ involvement such as CDI. Histiocytosis in the adult is a rare disease, predominantly seen with pulmonary involvement. Even more rarely, an isolated pituitary manifestation of histiocytosis is seen in the adult (28). Radiological procedures may be helpful in detecting histiocytosis; in any case only histology assures the diagnosis.

In cases 1 and 2 , in view of the patients' stable disease we found no opportunity to justify a biopsy of the pituitary. In the case of multi-organ involvement histological proof of extracerebral lesions is a valid diagnostic approach (cases 3-5).

Corticosteroids effectively suppress immunoreaction in neurosarcoidosis. Various adjuvant immunosuppressants have been discussed to minimise side effects of high dose steroid therapy, i.e. cyclosporine, azathioprine, methotrexate, cyclophosphamide, chlorambucil or radiation therapy (29). Pulse cyclophosphamide therapy appears to have good effects in adjuvant treatment of neurosarcoidosis (30). In multi-organ involvement of Wegener granulomatosis (WG) continuous cyclophosphamide treatment seems to have an advantage over intermittent therapy (12). However, a new multi-centre study demonstrated similar effectiveness, inducing first remission in generalised WG by both modalities of cyclophosphamide therapy (31). Patient 3 showed deterioration under steroid therapy alone, but a good remission with additional continuous cyclophosphamide over the course of twelve months. Remission or stable disease in all patients were measured by clinical evaluation, computed tomography and contrast enhanced brain MRI, revealing initial total remission in patients 4 and 5 (Figs 4 and 5), although pituitary dysfunction remained unchanged throughout the course of the disease.

To summarise, involvement of the endocrine system may account for the only presenting symptom of sarcoidosis. We have described five patients with this 
rare clinical presentation. Knowing that intracranial neurosarcoidosis may be correlated to a more malignant or even life threatening course of the disease (compare case 5) makes an intensive diagnosis and consequent therapy mandatory for preventing deterioration of sarcoidosis.

\section{References}

1 Chapelon C, Ziza JM, Piette JC, Levy Y, Raguin G, Wechsler B et al. Neurosarcoidosis: signs, course and treatment in 35 confirmed cases. Medicine 199069 261-276.

2 Oksanen V. Neurosarcoidosis: clinical presentations and course in 50 patients. Acta Neurologica Scandinavia 198673 283-290.

3 Stern BJ, Drumholz A, Johns C, Scott P \& Nissim J. Sarcoidosis and its neurological manifestations. Archives of Neurology 198542 909-917.

4 Graham E \& James DG. Neurosarcoidosis. Sarcoidosis 19885 125-131.

5 Ezzat S \& Josse RG. Autoimmune hypophysitis. Trends in Endocrinology and Metabolism 19978 74-80.

6 Thodou E, Asa SL, Kontogeorgos G, Kovacs K, Horvath E \& Ezzat S. Clinical case seminar: lymphocytic hypophysitis: clinicopathological findings. Journal of Clinical Endocrinology and Metabolism $1995802302-2311$

7 Pressman EK, Zeidman SM, Reddy UM, Epstein JI \& Brem H Differentiating lymphocytic adenohypophysitis from pituitary adenoma in the peripartum patient. Journal of Reproductive Medicine 199540 251-259.

8 Donald RA. The assessment of pituitary function. Clinical Biochemistry 199023 23-30.

9 Robertson GL. Diabetes insipidus. Endocrinology and Metabolism Clinics of North America 199524 549-572.

10 Fukata J, Shimizu N, Imura H, Hibi I, Tanaka K, Tanaka T et al. Human corticotropin-releasing hormone test in patients with hypothalamo-pituitary-adrenocortical disorders. Endocrine Journal 199340 597-606.

11 Vance ML. Hypopituitarism. New England Journal of Medicine 1994 $3301651-1662$.

12 Reinhold-Keller E, Kekow J, Schnabel A, Schmitt WH, Heller M, Beigel A et al. Influence of disease manifestation and antineutrophil cytoplasmatic antibody titer on the response to pulse cyclophosphamide therapy in patients with Wegener's granulomatosis. Arthritis and Rheumatism 199447 919-924.

13 Sharma OP \& Sharma AM. Sarcoidosis of the nervous system. A clinical approach. Archives of Internal Medicine $19911511317-$ 1321.

14 Bleisch VR \& Robbins SL. Sarcoid-like granulomata of the pituitary: a course of pituitary insufficiency. Archives of Internal Medicine $195289877-892$.

15 Chiang R, Marshall MC, Rosman PM, Hotson G, Mannheimer E \& Wallace EZ. Empty sella turcica in intracranial sarcoidosis. Pituitary insufficiency, primary polydipsia, and changing neuroradiologic findings. Archives of Neurology $198441662-665$.

16 Chapellan LJI, Olmedo LC, Martin JM, Marin MM, Villanueva MG, Zarza FM et al. Intrasellar mass with hypopituitarism as a manifestation of sarcoidosis. Journal of Neurosurgery 199073 283-286.

17 Féry F, Plat L, van de Borne P, Copgan E \& Mockel J. Impaired cointerregulation of glucose in a patient with hypothalamic sarcoidosis. New England Journal of Medicine 1999340 852-856.

18 Loh K-C, Green A, Dillon WP, Fitzgerald PA, Weidner N \& Tyrell JB. Diabetes insipidus from sarcoidosis confined to the posterior pituitary. European Journal of Endocrinology 1997137 514-519.

19 Mißler U, Mack M, Nowak G, Müller-Esch G, Reusche E, Borgis K-J et al. Pituitary sarcoidosis. Klinische Wochenschrift 199068 342345 .

20 Oksanen V, Grönhagen-Riska C, Fyhrquest F \& Somer H. Systemic manifestation and enzyme studies in sarcoidosis with neurologic involvement. Acta Medica Scandinavia 1985218 123-127.

21 Lawrence EC, Brousseau KP, Berger MB, Kurman CC, Marcon L \& Nelson DL. Elevated concentrations of soluble interleukin-2 receptors in serum samples and bronchoalveolar lavage fluids in active sarcoidosis. American Reviews of Respiratory Disease 1988 $137759-764$.

22 Lieberman J. Evaluation of serum angiotensin-converting-enzyme (ACE) level in sarcoidosis. American Journal of Medicine 197559 359-360.

23 Selroos OB. Biochemical markers in sarcoidosis. Critical Reviews in Clinical Laboratory Sciences 198624 185-216.

24 Strausz J, Müller-Quernheim J \& Ferlinz R. Sezernierter Interleukin-2-Rezeptor als Aktivitätsparameter der Sarkoidose. Deutsche Medizinische Wochenschrift 1989114 744-749.

25 Ziegenhagen MW, Benner UK, Zissel G, Zabel P, Schlaak M \& Müller-Quernheim J. Sarcoidosis: TNF- $\alpha$ release from alveolar macrophages and serum level of sIL-2R are prognostic markers. American Journal of Respiratory and Critical Care Medicine 1997 156 1586-1592.

26 Lexa F \& Grossmann RI. MR of sarcoidosis in the head and spine: spectrum of manifestations and radiographic response to steroid therapy. American Journal of Neuroradiology 199315 973982 .

27 Scott TF. Neurosarcoidosis: progress and clinical aspects. Neurology $1993438-12$.

28 Braumgartner I, Hochstetter Av, Baumert B, Luetolf U \& Follath F. Langerhans'-cell histiocytosis in adults. Medical and Pediatric Oncology 199728 9-14.

29 Agbogu BN, Stern BJ, Sewell C \& Yang G. Therapeutic considerations in patients with refractory neurosarcoidosis. Archives of Neurology 199552 875-879.

30 Lower EE, Broderick JP, Brott TG \& Baughman RP. Diagnosis and management of neurological sarcoidosis. Archives of Internal Medicine 1997157 1864-1868.

31 Guillevin L, Cordier J-F, Lhote F, Cohen P, Jarrousse B, Royer I et al. A prospective, multicenter, randomized trial comparing steroids and pulse cyclophosphamide in the treatment of generalised Wegener's granulomatosis. Arthritis and Rheumatism 199740 2187-2198.

Received 11 May 1999

Accepted 21 December 1999 